\title{
Case Report: Ischemic Enterocolitis Associated with Coronavirus Disease 2019: Two Case Reports and a Review of the Literature
}

\author{
Takeshi Kinjo, ${ }^{1 \star}$ Akira Hokama, ${ }^{2}$ Hideta Nakamura, ${ }^{1}$ Kazuya Miyagi, ${ }^{1}$ Yuri Higure, ${ }^{1}$ Mariko Otsuki, ${ }^{1}$ Naoya Nishiyama, ${ }^{1}$ \\ Masashi Nakamatsu, ${ }^{3}$ Tetsu Kinjo, ${ }^{2}$ Masao Tateyama, ${ }^{1}$ and Jiro Fujita ${ }^{1}$ \\ ${ }^{1}$ Department of Infectious, Respiratory, and Digestive Medicine, Graduate School of Medicine, University of the Ryukyus, Okinawa, \\ Japan; ${ }^{2}$ Department of Endoscopy, University of the Ryukyus Hospital, Okinawa, Japan; ${ }^{3}$ Infection Control Center, University of the \\ Ryukyus Hospital, Okinawa, Japan
}

\begin{abstract}
The COVID-19 pandemic has caused serious health and social concerns worldwide. Although the primary target of SARS-CoV-2 is the respiratory tract, SARS-CoV-2 infection also causes extrapulmonary symptoms. Previous articles have reported ischemic colitis in COVID-19 patients; however, information regarding its clinical manifestations and pathophysiology is limited. In this case report, we present two cases of ischemic enterocolitis in COVID-19 patients and review past case reports. Our literature review has shown that computed tomography rather than endoscopy was used for the diagnosis, and any region of the intestine was affected. Because the elevation of the D-dimer, which suggested a hypercoagulable state, was reported in most cases, we assumed that thrombosis at any level in the artery and vein was involved in the pathophysiology of COVID-19-associated enterocolitis. SARS-CoV-2-induced endotheliitis can cause both coarctation of the vessels and thrombosis; therefore, both patterns of ischemic colitis, occlusive and nonocclusive, may be involved in COVID-19-associated enterocolitis.
\end{abstract}

\section{INTRODUCTION}

COVID-19, first identified in Wuhan, China, in December 2019 , is the cause of an ongoing pandemic. Although the main manifestation of COVID-19 is respiratory infection, extrapulmonary symptoms, such as cerebral infarction, can occur. ${ }^{1}$ In addition, Pan et al. ${ }^{2}$ reported that $51 \%$ of COVID-19 cases had gastrointestinal symptoms such as loss of appetite, diarrhea, and vomiting. Recently, some articles have demonstrated ischemic colitis in COVID-19 patients; ${ }^{3-9}$ however, information on its clinical manifestations and pathophysiology is limited. Herein, we report two cases of COVID-19associated ischemic enterocolitis and review past case reports.

\section{CASE REPORTS}

Case 1. A 45-year-old previously healthy man visited a community hospital complaining of fever and dyspnea. The real-time PCR (QuantiTect Probe RT-PCR Kit, QIAGEN, Hilden, Germany) performed using the nasal swab was positive for SARS-CoV-2. His respiratory condition worsened, despite treatment with favipiravir. Thereafter, he was transferred to our hospital. He was intubated on day 3 of admission, and extracorporeal membrane oxygenation (ECMO) was initiated on day 5 . Together with ECMO therapy, unfractionated heparin was administered continuously. His condition gradually improved; ECMO was terminated on day 14, and he was weaned from ventilatory support on day 17 . However, he experienced hematochezia on day 18. Fecal cultures did not indicate the presence of pathological bacteria, and the tests for the Clostridioides difficile toxins $A / B$ were

\footnotetext{
*Address correspondence to Takeshi Kinjo, Department of Infectious, Respiratory, and Digestive Medicine, Graduate School of Medicine, University of the Ryukyus, 207 Uehara, Nishihara, Okinawa 903-0215, Japan. E-mail: t_kinjo@med.u-ryukyu.ac.jp
}

negative. In addition, the cytomegalovirus pp65 antigenemia assay was negative. Although endoscopy was abandoned, we suggested a diagnosis of ischemic enterocolitis owing to a persistent elevated D-dimer level (12.5 $\mu \mathrm{g} / \mathrm{mL}$ on day 18); unfractionated heparin administration was continued. Enhanced computed tomography (CT) on day 25 revealed no evidence of intravascular thrombi but showed layered thickening of the descending colon and engorgement of the mesenteric vessels, suggesting ischemic colitis (Figure 1). In addition, a thrombus in the left internal jugular vein, where the cannula was inserted during the ECMO therapy, was seen. Hematochezia improved upon the termination of tube feeding. The patient was switched from unfractionated heparin to an oral anticoagulant on day 33; he was discharged on day 38 . The use of the oral anticoagulant was continued for 6 months.

Case 2. A 68-year-old woman visited our hospital with a 2day history of intermittent left dorsal pain and fever. She had been hospitalized for COVID-19 pneumonia and was discharged with full recovery, 23 days previously. Abdominal CT on day 4 of this hospitalization revealed no evidence of intravascular thrombi but showed marked thickening with a target sign in the upper jejunum (Figure 2). The distribution of the lesion was segmental. The patient had not developed diarrhea during hospitalization, which suggested that infectious enterocolitis was not likely. Although she denied having melena, her blood hemoglobin had decreased from $11.4 \mathrm{~g} / \mathrm{dL}$ (on the day of this admission) to $8.5 \mathrm{~g} / \mathrm{dL}$ (on day 9 ); it increased to 9.9 $\mathrm{g} / \mathrm{dL}$ after discharge. During her previous hospital admission, her blood D-dimer level elevated to $23.7 \mu \mathrm{g} / \mathrm{mL}$ but decreased by $7.2 \mu \mathrm{g} / \mathrm{mL}$ at discharge. During this hospitalization, it reascended from 1.1 (day 1) to $4.5 \mu \mathrm{g} /$ $\mathrm{mL}$ (day 12), indicating a hypercoagulable state. Taken together, we diagnosed ischemic jejunitis associated with COVID-19. Her symptoms improved with conservative therapy such as bowel rest and pain killers. She was discharged on day 15. 


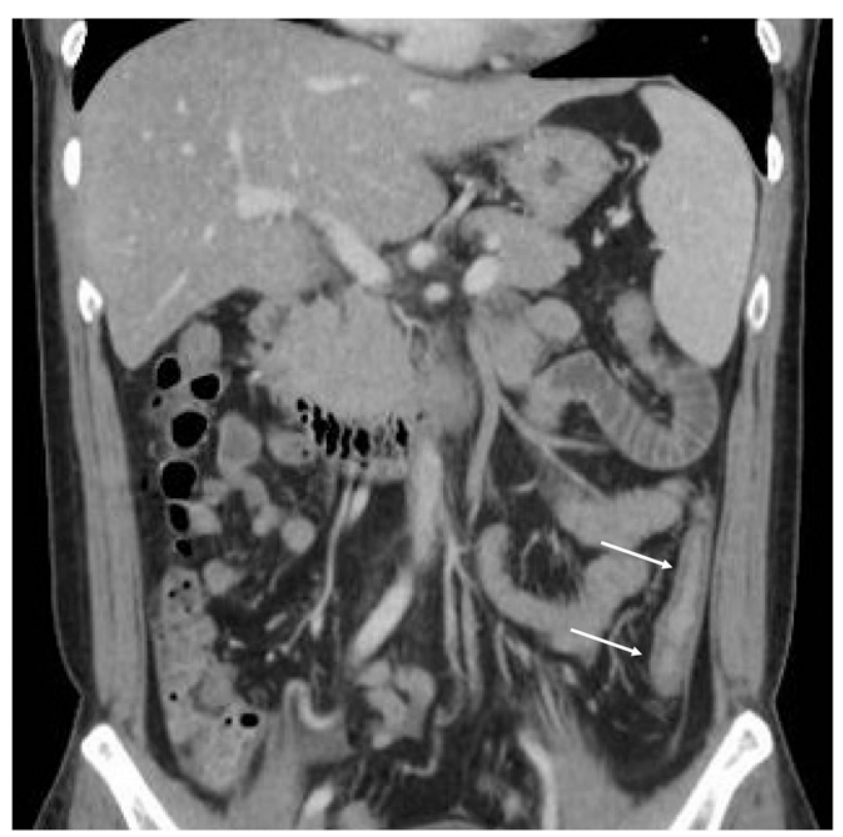

FIGURE 1. Coronal computed tomography (CT) scan of case 1. Enhanced CT scan on day 25 revealing a layered thickening of the descending colon (arrows) and engorgement of the mesenteric vessels.

\section{DISCUSSION}

SARS-CoV-2 infects host cells via the angiotensin-converting enzyme 2 expressed on type II alveolar cells and others, including endothelial cells. ${ }^{10}$ Systemic inflammation and diffuse endotheliitis by SARS-CoV-2 can induce a hypercoagulable state, resulting in arterial and venous thromboembolism. ${ }^{11,12}$ We

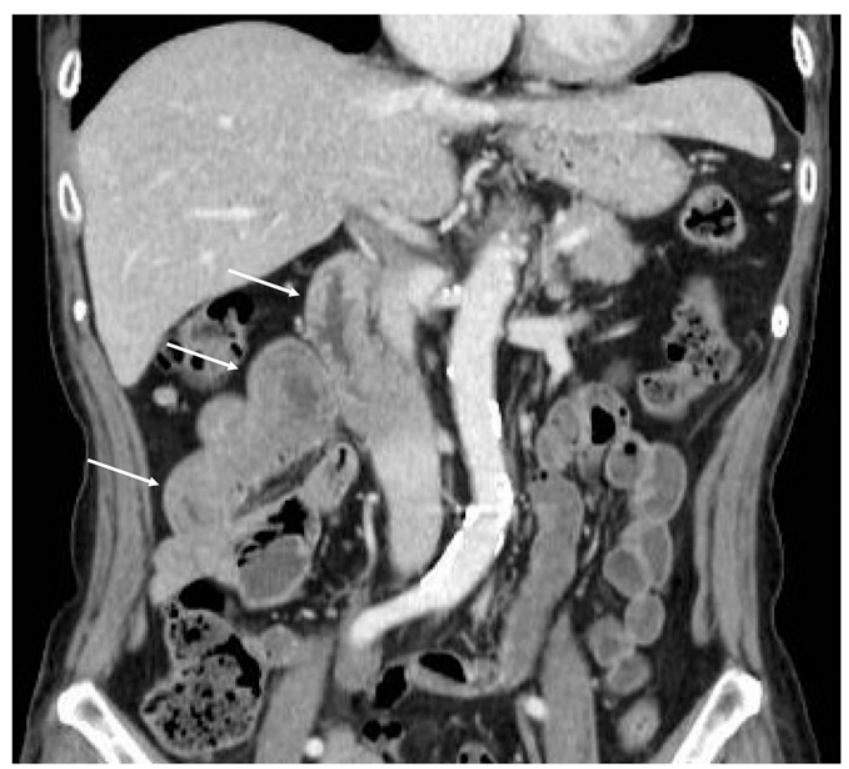

FIGURE 2. Coronal computed tomography (CT) scan of case 2. Enhanced CT scan on day 4 revealing a marked thickening with a target sign in the upper jejunum (arrows). The lesion is distributed segmentally, suggesting an ischemic rather than an infectious enterocolitis. reviewed past case reports in which COVID-19 patients, including our cases, presented with ischemic enterocolitis. These are shown in Table 1. CT was used for the diagnosis because endoscopy was abandoned in most cases. Endoscopy is an important tool for the diagnosis of gastrointestinal (Gl) diseases, including ischemic enterocolitis. However, in a realistic medical setting, the procedure is often abandoned in patients with COVID-19 because of a lack of personal protective equipment and the possible risk of SARS-CoV-2 transmission to healthcare workers. In terms of the sites of lesions in the past reports, any region of the intestine was affected.

Since the elevation of the D-dimer, which suggested a hypercoagulable state was reported in most cases, we assumed that thrombosis at any level in the artery and vein was involved in the pathophysiology of COVID-19-associated enterocolitis. Ischemic colitis is commonly categorized into two classical patterns, occlusive and nonocclusive. However, it is difficult to categorize COVID-19-associated ischemic enterocolitis into these two patterns because its pathophysiological mechanism is not fully understood. In fact, thrombi were observed during pathological examinations in cases where thrombi were not seen in CTs. ${ }^{5,7}$ We assumed that SARS-CoV-2-induced endotheliitis can cause both coarctation of the vessels and thrombosis; therefore, both patterns of ischemic colitis, occlusive and nonocclusive, may be involved in COVID-19associated enterocolitis.

In conclusion, our presentation of two cases of ischemic colitis in COVID-19 patients has indicated that we should be aware of systemic thrombotic complications, including ischemic enterocolitis, due to SARS-CoV-2 infection, as well as pulmonary manifestations. In addition, when developing their therapeutic strategies, physicians should consider COVID-19-related hypercoagulability when patients with COVID-19 experience GI bleeding. Because information regarding COVID-19-associated ischemic colitis is limited, the accumulation of more case reports and further research are needed to understand the pathophysiology.

Received August 26, 2020. Accepted for publication March 9, 2021. Published online March 18, 2021.

Acknowledgments: We acknowledge Editage (www.editage.com) for English language editing. Publication charges for this article were waived due to the ongoing pandemic of COVID-19.

Disclosure: Written informed consent was obtained from the patients for the publication of this case report. All authors met the ICMJE authorship criteria.

Authors' addresses: Takeshi Kinjo, Hideta Nakamura, Kazuya Miyagi, Yuri Higure, Mariko Otsuki, Naoya Nishiyama, Masao Tateyama, and Jiro Fujita, Department of Infectious, Respiratory, Graduate School of Medicine, University of the Ryukyus, Okinawa, Japan, E-mails: $t$ kinjo@med.u-ryukyu.ac.jp, hnhideta@gmail.com, miyagi.kazuya@ gmail.com, yr.1129.snvj@gmail.com, owan4x25@gmail.com, n.naoyaso@gmail.com, tateyama@med.u-ryukyu.ac.jp, and fujita@med.u-ryukyu.ac.jp. Akira Hokama and Tetsu Kinjo, Department of Endoscopy, University of the Ryukyus Hospital, Okinawa, Japan, E-mail: hokama-a@med.u-ryukyu.ac.jp and tetsu19761976@yahoo.co.jp. Masashi Nakamatsu, Infection Control Center, University of the Ryukyus Hospital, Okinawa, Japan, E-mail: massy@med.u-ryukyu.ac.jp.

This is an open-access article distributed under the terms of the Creative Commons Attribution (CC-BY) License, which permits unrestricted use, distribution, and reproduction in any medium, provided the original author and source are credited. 


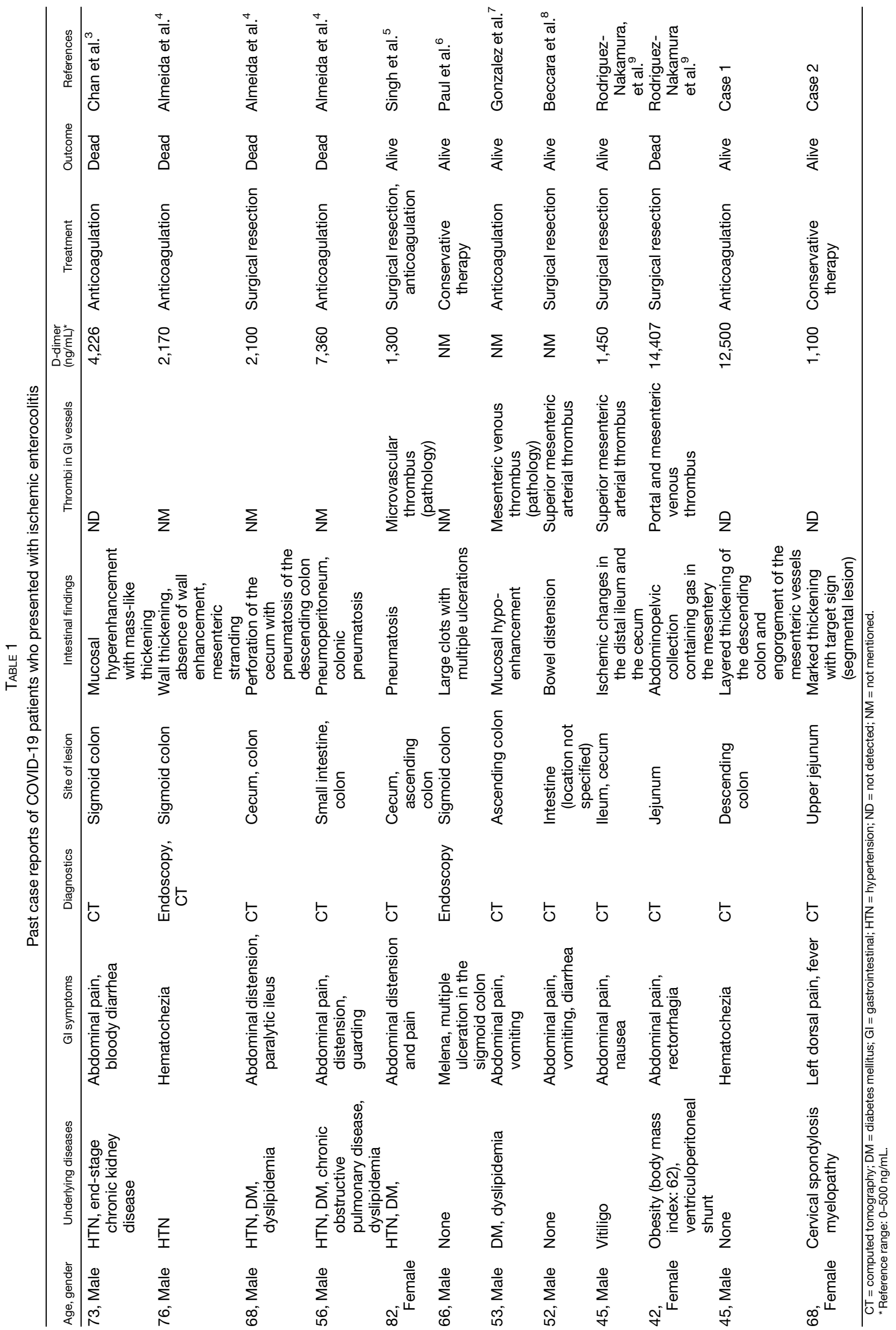




\section{REFERENCES}

1. Abobaker A, Raba AA, Alzwi A, 2020. Extrapulmonary and atypical clinical presentations of COVID-19. J Med Virol 92: 2458-2464.

2. Pan L et al., 2020. Clinical characteristics of COVID-19 patients with digestive symptoms in Hubei, China: a descriptive, crosssectional, multicenter study. Am J Gastroenterol 115: 766-773.

3. Chan KH, Lim SL, Damati A, Maruboyina SP, Bondili L, Abu Hanoud A, Slim J, 2020. Coronavirus disease 2019 (COVID-19) and ischemic colitis: an under-recognized complication. $A m \mathrm{~J}$ Emerg Med 38: 2758.e1-2758.e4.

4. Almeida Vargas A, Valentí V, Sánchez Justicia C, Martínez Regueira F, Martí Cruchaga P, Luján Colás J, Aliseda Jover D, Esteban Gordillo S, Cienfuegos JA, Rotellar Sastre F, 2020. Severe colon ischemia in patients with severe coronavirus-19 (COVID-19). Rev Esp Enferm Dig 112: 784-787.

5. Singh B, Mechineni A, Kaur P, Ajdir N, Maroules M, Shamoon F, Bikkina M, 2020. Acute intestinal ischemia in a patient with COVID-19 infection. Korean J Gastroenterol 76: 164-166.

6. Paul T, Joy AR, Alsoub H, Parambil JV, 2020. Case report: ischemic colitis in severe COVID-19 pneumonia: an unforeseen gastrointestinal complication. Am J Trop Med Hyg 104: 63-65.
7. González Lázaro P, Lomas Meneses A, Del Val Zaballos F, Morandeira Rivas A, 2020. Ischemic colitis and short bowel disease due to choronavirus disease 2019 (COVID 19). Clin Nutr ESPEN 40: 406-407.

8. Baccera LA, Pacioni C, Ponton S, Francavilla S, Cuzzoli A, 2020. Arterial mesenteric thrombosis as a complication of SARS-CoV-2 infection. Eur J Case Rep Intern Med 7: 001690.

9. Rodriguez-Nakamura RM, Gonzalez-Calatayud M, Martinez Martinez AR, 2020. Acute mesenteric thrombosis in two patients with COVID-19. Two cases report and literature review. Int J Surg Case Rep 76: 409-414.

10. Li W et al., 2003. Angiotensin-converting enzyme 2 is a functional receptor for the SARS coronavirus. Nature 426: 450-454.

11. Levi M, Thachil J, Iba T, Levy JH, 2020. Coagulation abnormalities and thrombosis in patients with COVID-19. Lancet Haematol 7: e438-e440.

12. Varga Z, Flammer AJ, Steiger $P$, Haberecker $M$, Andermatt $R$, Zinkernagel AS, Mehra MR, Schuepbach RA, Ruschitzka F, Moch $H, 2020$. Endothelial cell infection and endotheliitis in COVID-19. Lancet 395: 1417-1418. 